\title{
Crecimiento y reproducción del camarón Atya margaritacea (Decapoda: Atyidae) en el Río Presidio, Sinaloa, México
}

\author{
Jesús Sánchez Palacios, Rigoberto Beltrán Álvarez \& Juan Pedro Ramírez Lozano \\ Facultad de Ciencias del Mar, UAS, Apdo. Postal 610, Paseo Claussen s/n, Mazatlán, Sinaloa, México. Fax (69) \\ 82-86-56; jspmrm@yahoo.com
}

Recibido 01-III-2006. Corregido 06-VI-2007. Aceptado 31-X-2007.

\begin{abstract}
Growth and reproduction of the shrimp Atya margaritacea (Decapoda: Atyidae) in Río Presidio, Sinaloa, Mexico. Some growth and reproduction parameters of the population of the shrimp Atya margaritacea in Presidio River (Sinaloa, NW Mexico) were studied using 542 organisms collected with a Surber net in five sampling locations distributed from mid- to low river. Total lengths and gross weights ranged from 15 to 96 $\mathrm{mm}$ and 0.1 to $25.2 \mathrm{~g}$. The male:female ratio was $1.96: 1$ and males had larger sizes and weights. The relations of total length $(\mathrm{Lt})$ to gross weight $(\mathrm{Pt})$ were $P t=1.02 \times 10^{-5}(L t)^{3.2089}$ for males and $P t=2.29 \times 10^{-5}(L t)^{3.0159}$ for females. Growth in males was positively allometric whereas in females it was isometric. The regressions between total lengths and cephalothorax (Lc) were $\log L c=1.1118(\log L t)-0.6087$ for males and $\log L c=0.9945(\log L t)-0.4321$ for females. The relative growth between these body parts was allometrically positive in males and isometric in females; this result indicates a clear sexual dimorphism. Ovigerous females appeared in the rainy season (July to November). The absolute fecundity ranged from 1860 to 22400 eggs in females of 43 to $59 \mathrm{~mm}$ in length and 1.9 to $6.0 \mathrm{~g}$ in weight. The equations relating the number of eggs to length and weight were $\mathrm{Fec}=8.3 \times 10^{-7}(L t)^{5.8053}$ and $\mathrm{Fec}=732(\mathrm{Pt})^{1.836}$ respectively. Rev. Biol. Trop. 56 (2): 513-522. Epub 2008 June 30.
\end{abstract}

Key words: Atya margaritacea, Presidio River, allometric growth, sexual dimorphism, fecundity.

El río Presidio ocupa un importante lugar entre los ríos del estado de Sinaloa ubicado en la región noroeste de México. Hasta 1993 contaba sobre su curso con una presa derivadora, misma que fue destruida por las fuertes corrientes de agua provocadas por intensas lluvias que se registraron sobre su cuenca en ese año. Este hecho brindó una oportunidad de estudio de Atya margaritacea en el río sin obstáculos físicos.

En América A. margaritacea se encuentra distribuida a lo largo de la costa del Pacífico, desde La Paz, Baja California, México hasta el norte del Perú (Hobbs y Hart 1982, Holthuis 1986), habita en las zonas de los ríos con corrientes rápidas y alto contenido de oxígeno disuelto.

Se considera que esta especie juega un importante papel ecológico en la recuperación del sistema al remover los sedimentos y detritus después de una alta descarga (Pringle et al. 1993, Pringle 1996, March et al. 2002), además como consumidor de una gran variedad de detritus los transforma en energía disponible para los niveles tróficos superiores (Covich 1988).

Antecedentes acerca del estudio de los carideos del género Atya son escasos. MilneEdwards (1864) realizó una revisión de los crustáceos macruros y la familia de los Atyidae. Davant (1963) publicó una clave para la identificación de los camarones marinos y de río de importancia económica del oriente de Venezuela. Fryer (1977) reportó algunos aspectos de la morfología y funcionalidad ecológica de los camarones del género Atya en la República Dominicana, Hobbs y Hart (1982) revisaron aspectos taxonómicos de este género y March et al. (1998), Fièvet (1999) y Benstead 
et al. (2000) estudiaron el ciclo de vida y la migración de larvas de camarones anfídromos.

En México, Villalobos (1943), estudió las características morfológicas de A. scabra y en 1959 publicó una contribución al conocimiento de los carideos del país. Figueroa (1985) abordó algunos aspectos de la biología de $A$. margaritacea. Recientemente Martínez-Mayen y Román-Contreras (2000) encontraron que en el río Coyuca, en el estado de Guerrero, México, A. margaritacea se reproduce todo el año, acentuándose el fenómeno durante la época de lluvias. Los mismos autores señalan que la fecundidad de esta especie varia desde 1504 a 16200 huevos, la cual aumenta en función de la talla.

En Sinaloa, existen los reportes de la presencia de esta especie de Coronel et al. (1988) en el río Presidio, de Beltrán et al. (1994) en el río Baluarte y de Beltrán et al. (1996) y Campaña (1999) en el río Elota.

En el 2006 se iniciaron los trabajos de la construcción de la presa Picachos sobre el cauce del río presidio, lo que modificará sustancialmente las condiciones del mismo y consecuentemente el hábitat de esta y otras especies poniendo en riesgo su presencia en este sistema lótico. Debido a la importancia ecológica que $A$. margaritacea tiene y a la escasa información de su biología en los ríos del estado, el presente trabajo reporta los resultados sobre el tipo de crecimiento y algunos aspectos reproductivos para esta especie en este ecosistema acuático.

\section{MATERIAL Y MÉTODOS}

La recolecta de los organismos se realizó de enero a diciembre de 1995 en el Río Presidio, localizado en el estado de Sinaloa, México. La cuenca se ubica entre los $23^{\circ} 05^{\prime} 45^{\prime \prime}$ y $24^{\circ} 10^{\prime} 09^{\prime \prime} \mathrm{N}$ y entre los $105^{\circ}$ y $106^{\circ} 19^{\prime} 09^{\prime \prime}$ $\mathrm{W}$, cuenta con una superficie total de 7309.47 $\mathrm{km}^{2}$ y su longitud es de aproximadamente 125 $\mathrm{km}$. Se realizaron nueve muestreos de campo para lo cual se ubicaron cinco estaciones localizadas de la parte media hasta la desembocadura del río. Para la recolecta de los organismos se utilizó una red tipo Surber con $0.25 \mathrm{~m}^{2}$ de área de muestreo, en aguas rápidas con profundidades menores de $0.7 \mathrm{~m}$ y sustrato pedregoso. En cada sitio se efectuaron cuatro lances, removiendo manualmente las rocas dentro de la superficie de muestreo para que la corriente arrastrara a los organismos al fondo de la red.

Los organismos recolectados se colocaron en bolsas de plástico etiquetadas, poniendo especial atención en las hembras con huevos en incubación, las cuales se colocaron en bolsas individuales preservándose en formaldehído al $10 \%$ para su posterior análisis. En el laboratorio se midió la longitud total (Lt) y del cefalotórax (Lc) (mm), mediante un vernier, el peso total $(\mathrm{Pt})(\mathrm{g})$ se registró con una balanza digital. Los organismos fueron depositados en la colección de referencia del Laboratorio de Limnología y Ecología de Aguas Dulces de la Facultad de Ciencias del Mar y en la colección de referencia del Instituto de Ciencias del Mar y Limnología estación Mazatlán de la Universidad Autónoma de México con número de catalogo EMU-1085 (Hendricks 1994). La relación longitud-peso se determinó de acuerdo con la ecuación potencial $P t=A L t^{b}$ (Bagenal 1978), donde el valor de la constante b permite definir el tipo de crecimiento, considerándose como isométrico un valor de $\mathrm{b}=3$ y como alométrico cuando tiene un valor diferente (Ricker 1975) esta evaluación se realizó mediante la prueba t de Student. Así mismo la relación entre el cefalotórax y la longitud total se determinó según la ecuación alométrica $y=B x^{a}$ propuesta por Huxley (1924), transformada a términos de logaritmos, tomando la forma $\log y=\log a+b \log x$ (Clayton y Snoweden 1991) en esta relación se considera crecimiento isométrico si $b=1$ y como alométrico si es diferente, tal evaluación se realizó mediante la prueba t de Student. El sexo se determinó de manera visual, para evaluar la desviación de la proporción sexual esperada 1:1 se utilizó la prueba $\chi^{2}$. Para la madurez sexual se tomó en cuenta únicamente a las hembras (debido a la facilidad para observar el desarrollo de las gónadas a través del cefalotórax o bien para detectar la presencia de huevos portados en los 
pleópodos), misma que se estableció basándose en la escala de madurez gonadal propuesta por Guzmán (1987) para Macrobrachium tenellum. La fecundidad se obtuvo mediante el método gravimétrico (Bagenal 1978).

\section{RESULTADOS}

Estructura de la población: en total se capturaron 542 organismos de A. margaritacea, con una variación de longitud total en machos de 25 a $96 \mathrm{~mm}$ con promedio de $60.5 \pm 15.4 \mathrm{~mm}$, y en hembras de 22 a $65 \mathrm{~mm}$ con promedio de $46.4 \pm 9.1 \mathrm{~mm}$, mientras que en indiferenciados se registraron de 15 a $32 \mathrm{~mm}$ con promedio de $22.3 \pm 5.1 \mathrm{~mm}$. Con respecto al peso, en machos fluctuó entre 0.3 a $25.2 \mathrm{~g}$ con un promedio de
$7.02 \pm 5.1 \mathrm{~g}$, mientras que en hembras fue de 0.3 a 7 g con promedio de $2.7 \pm 1.4$ g. y en indiferenciados fue de 0.1 a $0.7 \mathrm{~g}$ con promedio de $0.3 \pm 0.2 \mathrm{~g}$. La distribución de frecuencias de longitud de machos y hembras fue diferente (prueba para dos muestras independientes de Kolmogorov-Smirnov; $\mathrm{D}=0.5353, \mathrm{p}<0.001$ ). Los machos fueron significativamente mayores que las hembras (Prueba U de Mann-Whitney; $\mathrm{U}=11358, \mathrm{p}<0.0001)$ y el $97.4 \%$ de los organismos $>60 \mathrm{~mm}$ fueron machos (Fig. 1).

Relaciones biométricas: en la relación longitud total-peso total (Fig. 2), se encontró que el modelo que mejor la describe fue el potencial. En machos la ecuación fue $P t=1.09 \times 10^{-5}(L t)^{3.2089}, r=0.9942$, para

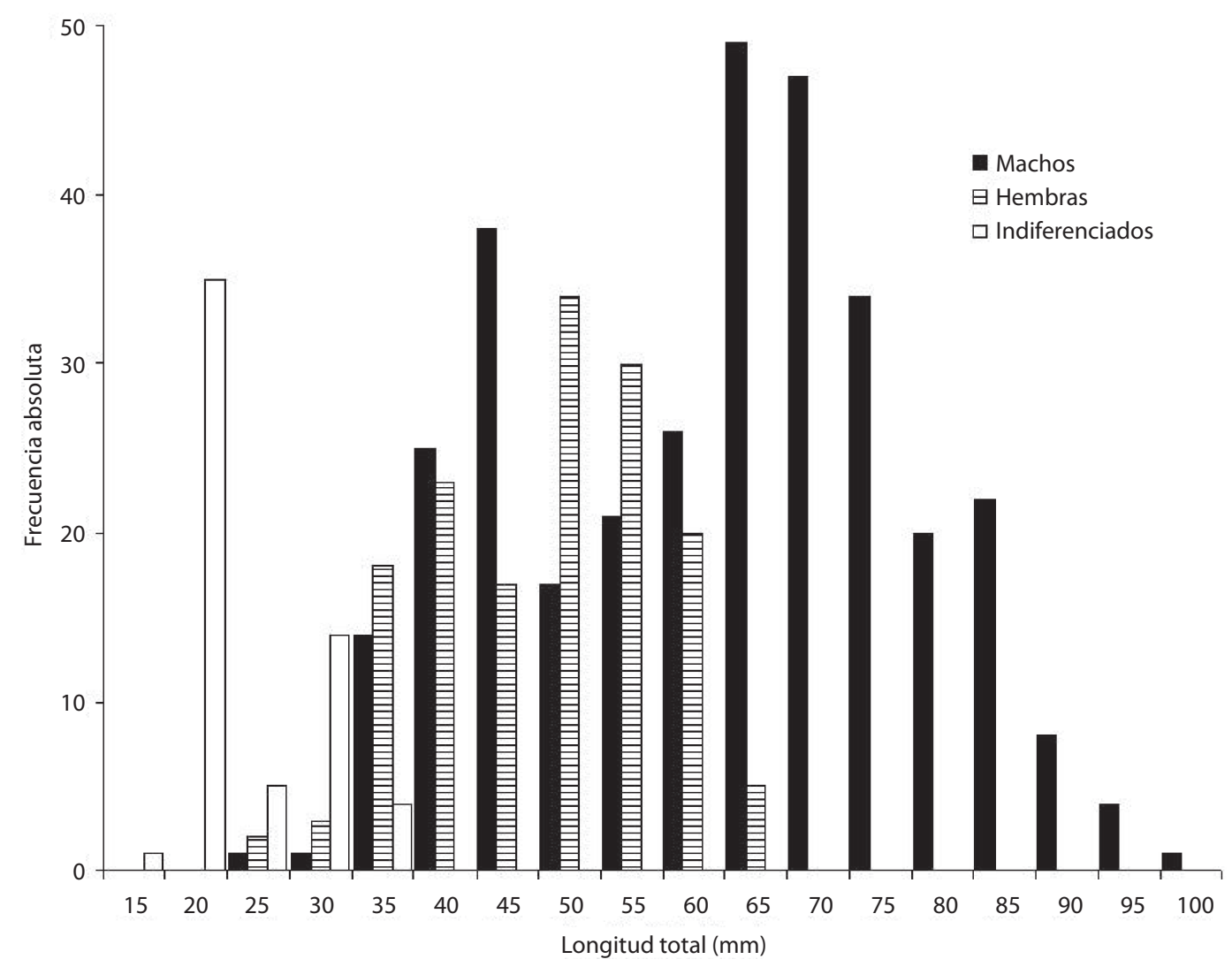

Fig. 1. Distribución de tallas de Atya margaritacea por sexo.

Fig. 1. Size distribution, by sex, for Atya margaritacea. 


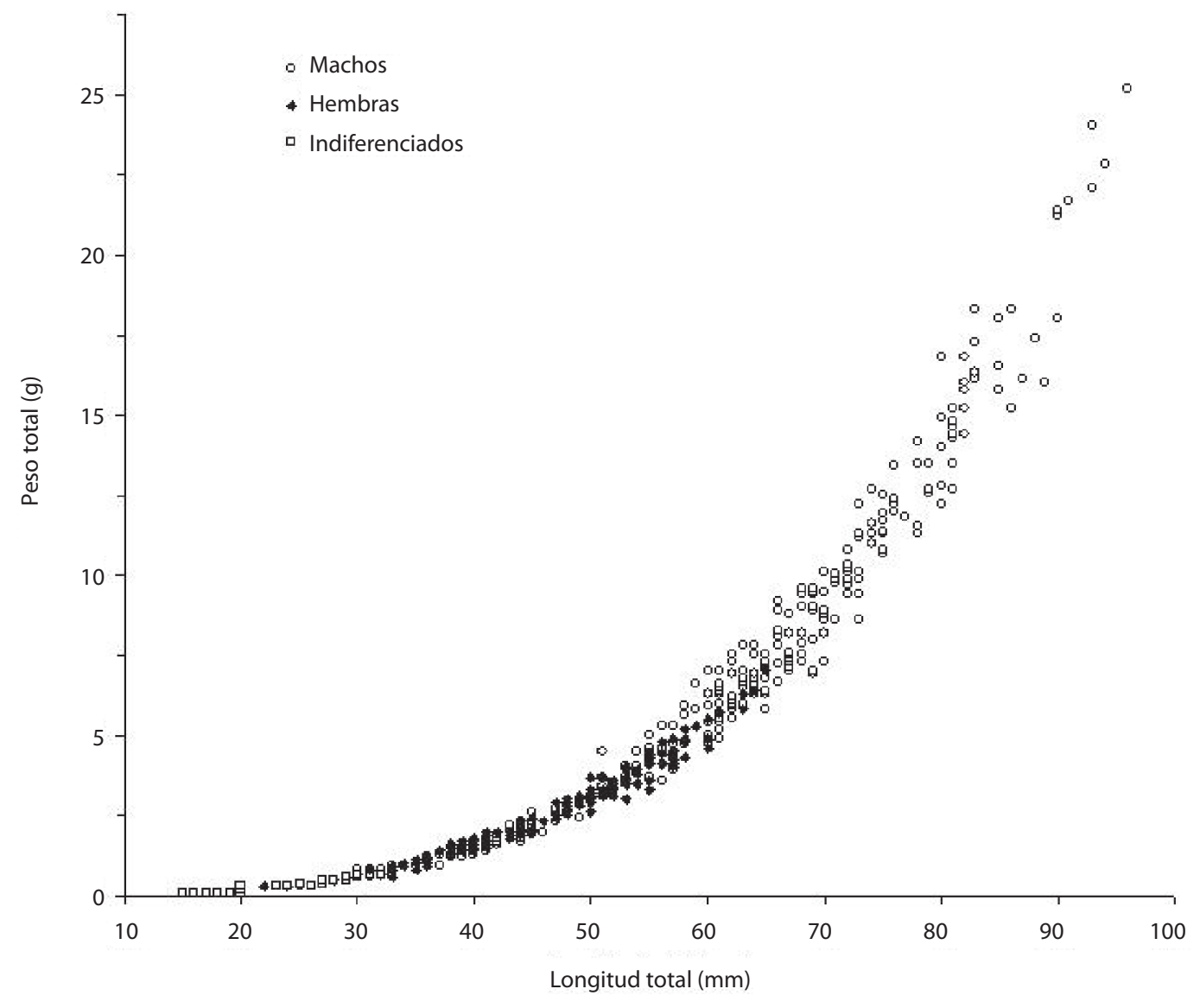

Fig. 2. Relación longitud - peso de Atya margaritacea.

Fig. 2. Length-weight relationships for Atya margaritacea.

hembras fue $P t=2.29 \times 10^{-5}(L t)^{3.0159}, r=0.9904$ y los indiferenciados $P t=2.28 \times 10^{-6}(L t)^{3.3089}$, $r=0.9457$ las correlaciones anteriores fueron altamente significativas $(\mathrm{p}<0.001)$. Se encontraron diferencias entre las pendientes de las relaciones de longitud-peso por sexo siendo mayor la de machos a la de hembras (ANCOVA; $F=22.5962, p<0.0001$ ). En machos la pendiente $(b=3.2089)$ de la ecuación anterior fue significativamente mayor del valor teórico de tres (prueba $\mathrm{t}=10.8728, \mathrm{p}<0.0001$ ), indicando que el crecimiento para este sexo fue del tipo alométrico positivo, en cambio en hembras la pendiente $(b=3.0159)$, no resultó diferente del valor teórico (prueba $\mathrm{t}=0.4628, \mathrm{p}=0.3221$ ), por lo que el crecimiento se consideró como isométrico.

Relación longitud total-longitud cefalotórax: en machos esta relación se explicó por el modelo $\log L c=1.1118$ $(\log L t)-0.6087, r=0.9938$, en hembras $\log L c=0.9945(\log L t)-0.4321, r=0.9845$ y $\log L c=1.0863(\log L t)-0.5747, r=0.9513$ para indiferenciados. El análisis de covarianza indicó diferencias significativas en las pendientes de estas relaciones entre sexos resultando mayor la de machos a la de hembras $(\mathrm{F}=40.943, \mathrm{p}<0.0001)$. En machos la pendiente $(b=1.1118)$, fue significativamente mayor a la unidad (prueba $\mathrm{t}=16.3328, \mathrm{p}<0.0001$ ). lo que 
permitió establecer el crecimiento como alométricamente positivo, en tanto que en hembras la pendiente $(b=0.9945)$ no fue diferente del valor teórico de uno (prueba $\mathrm{t}=0.3824, \mathrm{p}=0.3514$ ), considerado para un crecimiento isométrico.

Composición sexual: de los 539 organismos recolectados $330(60.9 \%)$ fueron machos, $153(28.2 \%)$ hembras y $59(10.9 \%)$ indiferenciados (Fig. 3). La proporción macho-hembra fue de 1.96:1 la cual fue significativamente diferente de la relación teórica de 1:1 (prueba $\left.\chi^{2}=64.865, \mathrm{gl}=1, \mathrm{p}<0.0001\right)$.

Madurez sexual: la madurez sexual se analizó a partir de la aparición, en julio, de las primeras hembras con huevos en incubación (fase III) las cuales alcanzaron el 90.3\% del total de organismos colectados (Cuadro 1), en los siguientes meses, y hasta noviembre, el porcentaje de hembras ovígeras representan
CUADRO 1

Madurez sexual (\%) de Atya margaritacea hembras

TABLE 1

Sexual maturity (\%) for Atya margaritacea females

\begin{tabular}{lcccc} 
& \multicolumn{5}{c}{ Fases de } & Madurez \\
Mes & I & II & III & IV \\
Jul & 3.2 & 0 & 90.3 & 6.5 \\
Ago & 0 & 0 & 100 & 0 \\
Sep & - & - & - & - \\
Oct & 0 & 0 & 85.8 & 14.2 \\
Nov & 0 & 0 & 86.6 & 13.4 \\
Dic & 9.4 & 9.4 & 28.2 & 53.0
\end{tabular}

más de $85 \%$ y el resto fueron hembras que concluyeron la incubación de los huevos (fase IV). $\mathrm{El}$ inicio del periodo reproductivo coincide con la presencia de las primeras lluvias en la región.

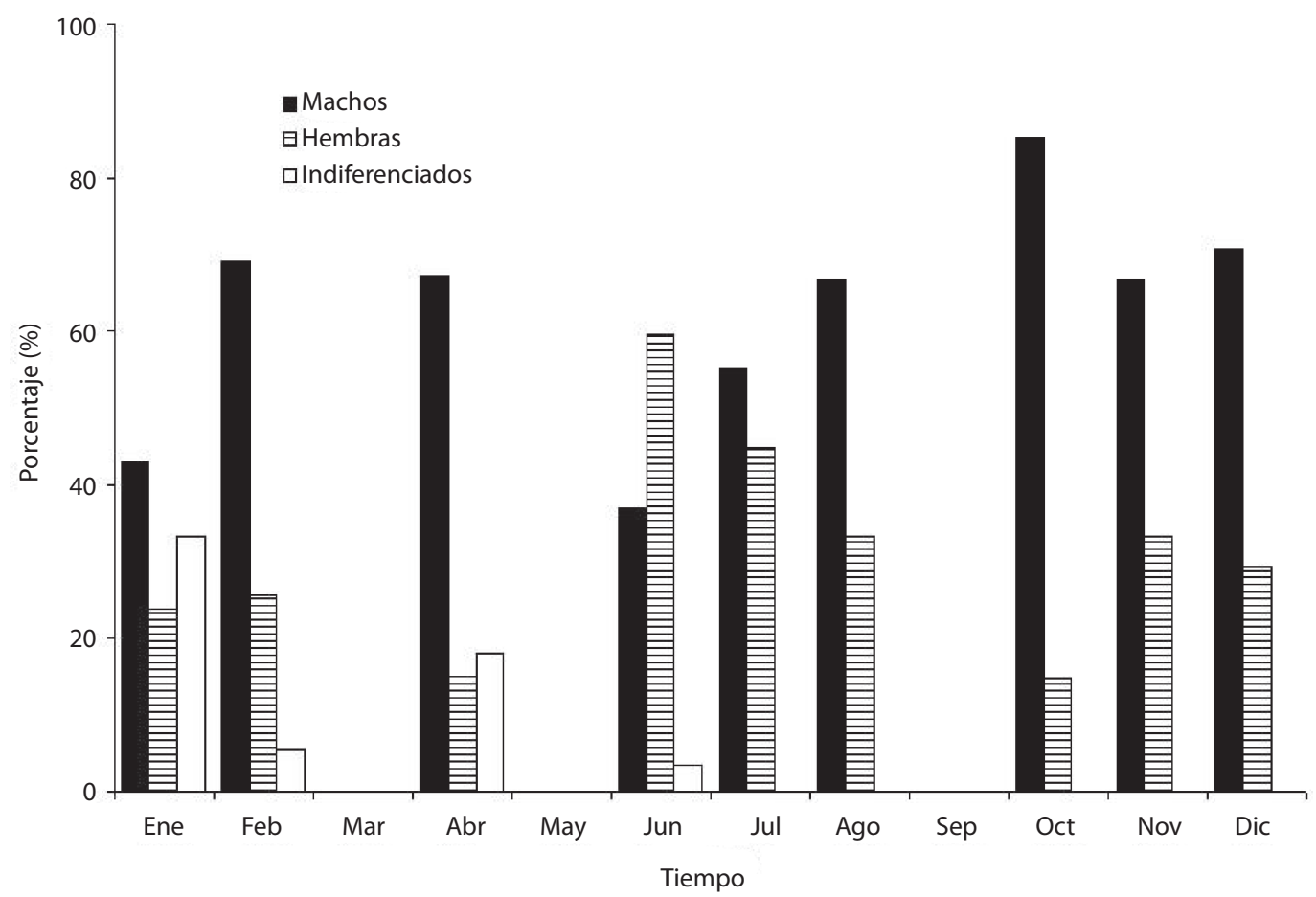

Fig. 3. Variación mensual de la proporción sexual de Atya margaritacea.

Fig. 3. Monthly variation of sex proportion for Atya margaritacea. 
Para diciembre el porcentaje de hembras maduras disminuye, al mismo tiempo que las hembras en post-reproducción (fase IV) se vuelven dominantes. Simultáneamente aparecen las fases I (juveniles) y II (pre-reproducción).

La hembra de menor talla en maduración registró $35 \mathrm{~mm}$ de longitud total y $1.1 \mathrm{~g}$ de peso, así mismo la hembra ovígera de menor tamaño tuvo $40 \mathrm{~mm}$ y $1.5 \mathrm{~g}$ de longitud total y peso respectivamente.

Fecundidad: la capacidad reproductiva para la especie se estableció a partir de la fecundidad absoluta de 41 hembras en fase III de maduración sexual. Se encontró que la fecundidad varió de 1860 a 22400 huevos en hembras de 43 y $59 \mathrm{~mm}$ de longitud total y 1.9 y $6 \mathrm{~g}$ de peso respectivamente. Al relacionar la fecundidad tanto con la longitud (Fig. 4), como con el peso (Fig. 5) se encontró que el modelo que mejor relaciona estas variables fue el potencial expresadas por $\mathrm{Fec}=8.3 \times 10^{-7}$ $(L t)^{5.8053}, r=0.8018$ para longitud y $\mathrm{Fec}=732$ $(P t)^{1.836}, r=0.8339$ para peso, ambas ecuaciones altamente significativas $(\mathrm{p}<0.0001)$.

\section{DISCUSIÓN}

En este estudio se constata que A. margaritacea habita una amplia extensión del río Presidio, en sitios donde sus principales características son agua con corriente rápida muy oxigenada, con poca profundidad y fondo constituido mayormente por canto rodado. La

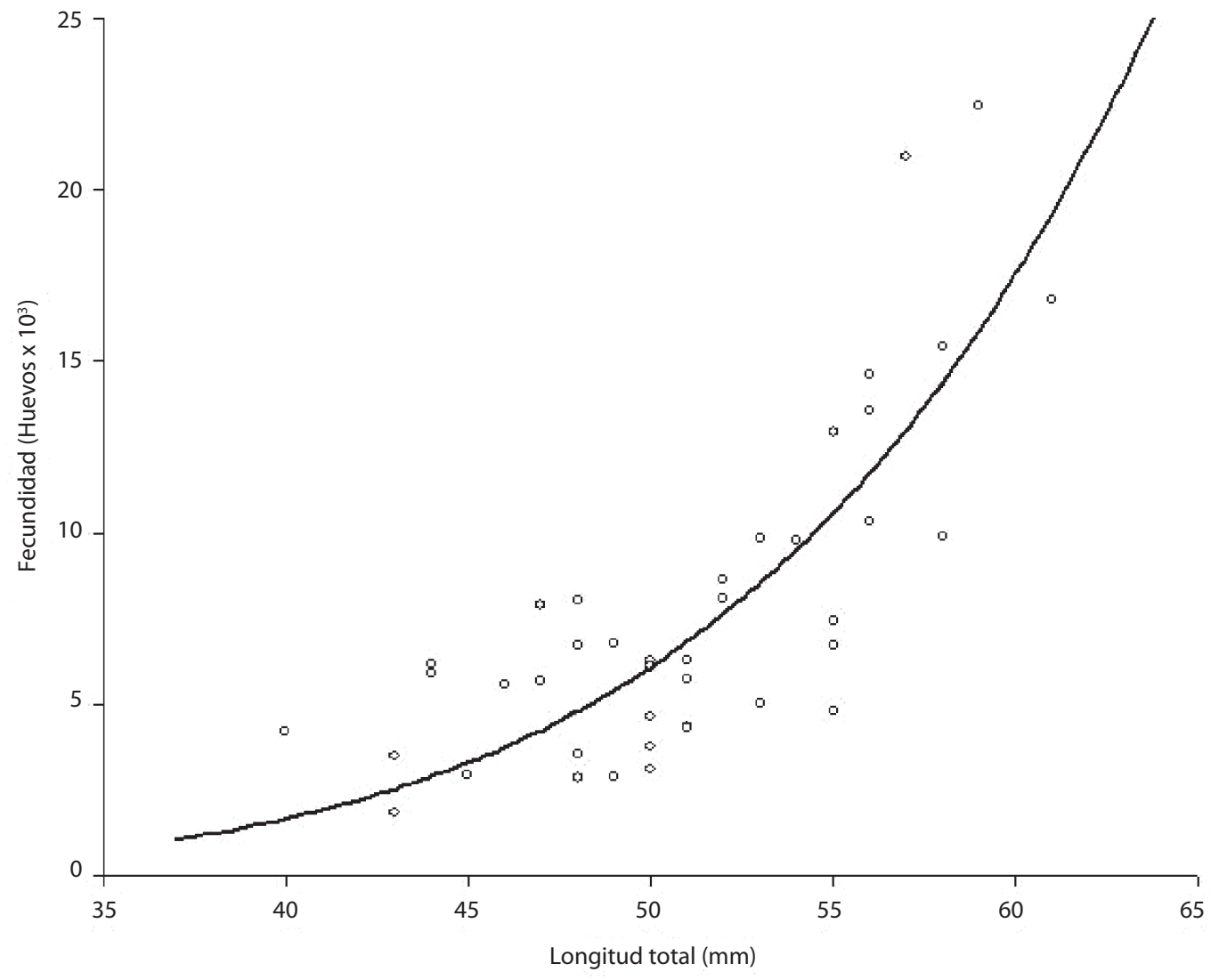

Fig. 4. Relación fecundidad-longitud de Atya margaritacea.

Fig. 4. Fecundity-length relationships for Atya margaritacea. 


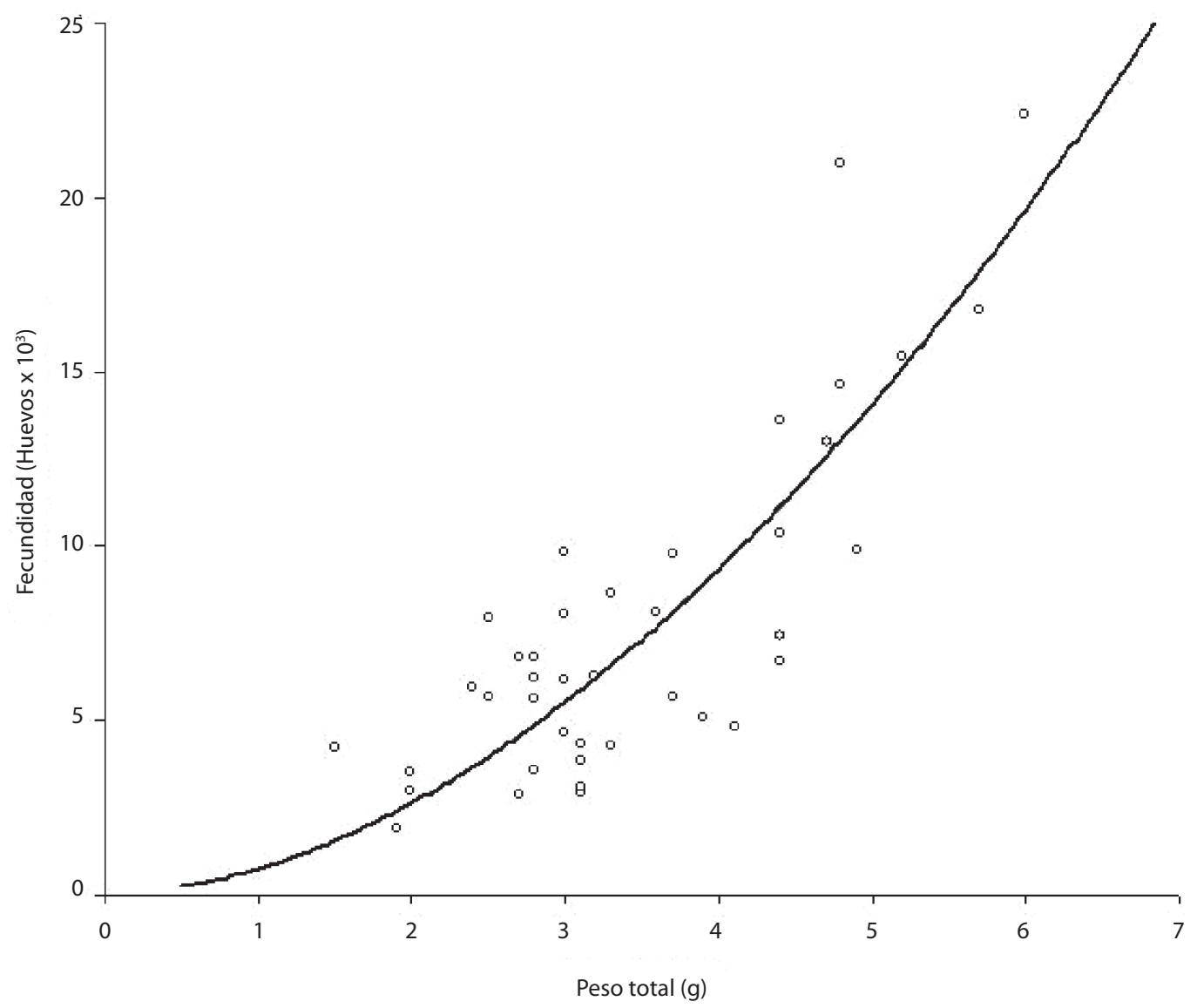

Fig. 5. Relación fecundidad-peso de Atya margaritacea.

Fig. 5. Fecundity-weight relationships for Atya margaritacea.

longitud total máxima de $A$. margaritacea encontrada en este estudio ( $96 \mathrm{~mm}$ ) fue mayor a la reportada por Hobbs y Hart (1982) (94 $\mathrm{mm}$ ), pero menor a la de $98.5 \mathrm{~mm}$ medida por Martínez-Mayen et al. (2000).

Existe un claro dimorfismo sexual en $A$. margaritacea en organismos mayores de $45 \mathrm{~mm}$ de longitud total ya que los machos alcanzan mayores tallas que las hembras, lo que permite separarlos visualmente. A este respecto, Hartnoll (1982) menciona que el crecimiento en crustáceos es similar en ambos sexos hasta que alcanzan la madurez, a partir de la cual el crecimiento en las hembras puede ser menor debido a la producción e incubación de los huevos. Diferencias en las tallas entre sexos han sido reportada por Cubillas-Hernández et al. (1989) en $A$. scabra, especie cercana a A. margaritacea y en particular para A. margaritacea por Hobbs y Hart (1982) y Martínez-Mayen et al. (2000).

La relación entre la longitud y el peso permite establecer el tipo de crecimiento que presentan los organismos (Ricker 1975). En este sentido el crecimiento alométrico ha sido reportado en crustáceos como Macrobrachium iheringi (Ortmann, 1897) por Lobao et al. (1986) y en Macrobrachium acanturus (Weigmann, 1836) por Roverso et al. (1993). En el presente estudio el crecimiento de machos en A. margaritacea fue alométrico positivo en tanto que en hembras fue isométrico lo cual coincide con lo encontrado por Martínez-Mayen et al. (2000). 
Respecto a la relación entre la longitud total y longitud del cefalotórax, la pendiente de la ecuación en machos indicó un crecimiento relativo de tipo alométrico positivo en tanto que en hembras fue isométrico, similar a lo encontrado por Martínez-Mayen et al. (2000). Por otro lado se encontraron diferencias significativas en las pendientes de la relación entre estas partes del cuerpo entre sexos, observándose que el cefalotórax de los machos es proporcionalmente mayor que en las hembras. Además, es de señalar que la diferencia en las tendencias de las relaciones biométricas coincide con la talla de la hembra madura de menor tamaño encontrada, lo que implica un claro dimorfismo sexual.

Los machos fueron más abundantes que las hembras en gran parte del año a excepción del mes en que se presentan las lluvias (junio), cuando la relación se invierte a favor de las hembras, en julio, la presencia de ambos sexos prácticamente se iguala. Lo anterior refleja una relación estrecha entre la reproducción y la época de lluvias, lo que puede obedecer a una estrategia reproductiva de la especie, o bien a un probable aumento en la vulnerabilidad de las hembras al arte de pesca utilizadas en su captura. En este sentido Figueroa (1985), señala que las hembras son más abundantes sólo en agosto, mientras que Martínez-Mayen y Román-Contreras (2000), encuentran una preponderancia de hembras en abril, mayo y septiembre.

La recolecta de hembras ovígeras a partir de julio permite establecer que las hembras de A. margaritacea en este río se reproducen al registrarse las primeras lluvias y continúa el resto del año, apreciándose que los organismos alcanzan su madurez sexual a los $35 \mathrm{~mm}$ de longitud total. En este sentido Hobbs y Hart (1982), afirman que esta especie inicia la reproducción a tallas de aproximadamente $22 \mathrm{~mm}$ de longitud total, mientras que Martínez-Mayen y RománContreras (2000) reportan que en el río Coyuca, Guerrero, México, se reproduce todo el año y alcanza su madurez a los $32 \mathrm{~mm}$ de longitud total participando más activamente en la reproducción cuando alcanza entre 45 y $54 \mathrm{~mm}$.
Se observó una amplia variación en el número de huevos en organismos de una misma longitud y peso, una posible explicación es que al manipular las hembras grávidas, no obstante el especial cuidado con que se realizaba su captura, se pierde parte de los huevos, sobre todo en los de avanzada madurez. El máximo número de huevos en incubación contabilizados fue de 22400 en una hembra de $59 \mathrm{~mm}$ de longitud total y $6 \mathrm{~g}$ de peso, lo cual es superior a lo reportado por Figueroa (1985) que obtiene 12572 huevos en una hembra de $16.5 \mathrm{~mm}$ de longitud del caparazón y a los 16200 huevos encontrado por Martínez-Mayen y RománContreras (2000) en una hembra de $66 \mathrm{~mm}$ de longitud total.

Se ha documentado ampliamente el efecto que tiene sobre la biota la construcción embalses sobre los ríos los cuales constituyen una barrera que limita el desplazamiento particularmente de peces y camarones anfídromos como los del género Atya, a tal grado que se reporta su ausencia casi total en ríos corriente arriba de las represas (Benstead et al. 1999, Pringle et al. 2000, Fièvet et al. 2001a, March et al. 2002). En este sentido la construcción de la presa Picachos sobre el río Presidio es de esperar modifique el cauce de éste, limitando el libre desplazamiento A. margaritacea lo que pondrá en riesgo su existencia, principalmente en la cuenca del rió superior a las aguas del embalse.

\section{AGRADECIMIENTOS}

Los autores expresan su agradecimiento a Librada Sánchez Osuna por su valiosa ayuda en la identificación de los organismos y a Salvador Organista Sandoval por sus comentarios al manuscrito.

\section{RESUMEN}

Se estudiaron el crecimiento y reproducción de de Atya margaritacea, con base en 542 organismos capturados en cinco sitios distribuidos desde la parte media de la cuenca hasta la desembocadura del río Presidio (Sinaloa, NW de México). La recolecta se realizó mediante el empleo de una red Surber, recolectándose organismos cuya longitud total y peso total variaron de 15 a $96 \mathrm{~mm}$ y 0.1 a 
$25.2 \mathrm{~g}$ respectivamente. La proporción macho:hembra fue 1.96:1 y los machos alcanzaron las mayores tallas y pesos. La relación entre la longitud total $(L t)$ y el peso total $(P t)$ se estableció mediante el modelo $P t=1.02 \times 10^{-5}(L t)^{3.2089}$ para los machos y por $P t=2.29 \times 10^{-5}(L t)^{3.0159}$ para las hembras. El crecimiento en machos resultó alométricamente positivo mientras que en hembras fue isométrico. La regresión entre la longitud total y longitud cefalotórax ( $L c)$ fue $\log L c=1.1118(\log L t)-0.6087$ en machos y $\log L c=0.9945(\log L t)-0.4321$ en hembras. El crecimiento relativo entre estas partes del cuerpo resultó alométrico positivo en machos e isométrico en hembras, indicando un claro dimorfismo sexual de estos organismos. Hembras ovígeras se presentaron en la época de lluvias (julio a noviembre). La fecundidad absoluta varió de 1860 a 22400 huevos en hembras de 43 y $59 \mathrm{~mm}$ de longitud y de 1.9 a $6.0 \mathrm{~g}$ de peso. La ecuación que relacionó el número de huevos con la longitud y peso, fue $\mathrm{Fec}=8.3 \times 10^{-7}$ $(L t)^{5.8053}$ y $\mathrm{Fec}=732(P t)^{1.836}$ respectivamente.

Palabras clave: Atya margaritacea, Río Presidio, crecimiento alométrico, dimorfismo sexual, fecundidad.

\section{REFERENCIAS}

Bagenal, B.T. 1978. Aspects of fish fecundity, p. 75-101. In S.D. Gerking (ed.). Ecology of Freshwater Fish Production. Blackwell Scientific, Oxford, Inglaterra.

Beltrán, A., J. Sánchez, G. Arroyo \& J.P. Ramírez. 1994. Informe final del proyecto "Estudio Hidrobiológico del río Baluarte, previo al embalsamiento de sus aguas por la construcción de la derivadora el Tamarindo, Sinaloa, México". UAS-SEP, Mazatlán, México.

Beltrán, A., J. Sánchez, G. Arroyo \& J.P. Ramírez. 1996. Informe final del estudio Hidrológico del río Elota, Sin. México. UAS-SEP, Mazatlán, México.

Benstead, J.P., J.G. March \& C.M. Pringle. 2000. Estuarine larval development and upstream postlarval migration of freshwater shrimps in two tropical rivers of Puerto Rico. Biotropica 32: 545-548

Benstead, J.P., J.G. March, C.M. Pringle \& F.N. Scatena. 1999. Effects of a low-head dam and water abstraction on migratory tropical stream biota. Ecological Applications: 9: 656-668.

Campaña, T.A. 1999. Aspectos biológicos de los carideos del río Elota, Sinaloa, México. Tesis Licenciatura, Facultad de Ciencias del Mar, Universidad Autónoma de Sinaloa, Sinaloa, México.

Clayton, D.A. \& R.J. Snowden. 1991. Allometric growth in Ilyoplax stevensi (Decapoda, Brachiura, Ocypodidae). Crustaceana 61: 1-10.
Coronel, V.J., C.H. Vargas, B.C. López \& T.H. Nordhal. 1988. Contribución al análisis biológico de las poblaciones de camarones de agua dulce (Macrobrachium spp. y Atya margaritacea) en la presa derivadora Siqueros, Sinaloa, México. Tesis de Licenciatura, Facultad de Ciencias del Mar, Universidad Autónoma de Sinaloa, Sinaloa, México.

Covich, A.P. 1988. Atyid shrimp in the headwaters of the Luquillo Mountains, Puerto Rico: filter feeding in the natural and artificial streams. Verhandl. Intern. Vereinigung Theor. Angew. Limnol. 23: 2108-2113.

Cubillas-Hernández L.F., G. Benítez \& Z. Chávez. 1989. Aspectos de la población de Atya scabra (Leach) en el río La Antigua, Veracruz, México. Resúmenes $\mathrm{X}$ Congreso Nacional de Zoología, México D.F., México.

Davant, P. 1963. Clave para la identificación de los camarones marinos y de río con importancia económica en el oriente de Venezuela. Instituto Oceanográfico, Universidad de Oriente, Sucre, Venezuela.

Figueroa, L.G. 1985. Algunos aspectos de la biología de Atya margaritacea (Decapoda, Atyidae). Tesis profesional, Escuela Nacional de Ciencias Biológicas, I.P.N., México D.F., México.

Fièvet, E. 1999. An experimental survey of freshwater shrimp upstream migration in an impounded stream of Guadeloupe Island, Lesser Antilles. Archiv Hydrobiol. 144: 339-355.

Fièvet, E.S., S. Dolodec \& P. Lim. 2001a. Distribution of migratory fishes and shrimps along multivariate gradients in tropical island streams. J. Fish Biol. 59: 390-402.

Fryer, G. 1977. Studies on the functional Morphology and Ecology of the Atyid prawns of Dominica. Phi. Trans. Royal Soc. London 277: 57-129.

Guzmán, A.M. 1987. Biología, Ecología y Pesca del langostino Macrobrachium tenellun (Smith, 1881), en las lagunas costeas del estado de Guerrero, México. Tesis Doctoral, Instituto de Ciencias del Mar y Limnología, UNAM, México D.F., México.

Hartnoll, R.G. 1982. Growth, p 111-196. In D.E. Bliss (ed.). The Biology of Crustacea: Embryology Morphology and Genetics. Academic, New York, EEUU.

Hendrickx, M.E. 1994. Catálogo de crustáceos estomatópodos y decápodos. Colección de Referencia, Estación Mazatlán, ICML, UNAM. Comisión Nacional para el Conocimiento y Uso de la Biodiversidad e Instituto de Ciencias del Mar y Limnología, UNAM, México D.F., México. 
Hobbs, H.H. \& C.W. Hart. 1982. The shrimp genus Atya (Decapoda: Atyidae). Smit. Contri. Zool. 364: 1-143.

Holthuis, L.B. 1986. Fresh-water shrimps of the family Atyidae (Crustacea: Decapoda) from western Colombia. J. Crust. Biol. 6: 438-445.

Huxley, J.S. 1924. Constant differential growth-ratios and their significance. Nature 114: 895.

Lobao, V.L., M.R.Z.N. Musto, N.E.T. Rojas, M. Lace \& M.F.S. Magalhaes. 1986. Estudo populacional de Macrobrachium iheringi (Ortmann, 1897) (Decapoda, Palaemonidae) do Rió Buava-SP. Bol. Inst. Pesca 13: 37-43.

March, J.G., J.P. Benstead, C.M. Pringle \& F.N. Scatena. 1998. Migratory drift of larval freshwater shrimps in two tropical streams, Puerto Rico. Freshwater Biol. 40: $261-273$

March, J.G., C.M. Pringle, M.J. Townsend \& A.I. Wilson. 2002. Effects of freshwater shrimp assemblages on benthic communities along an altitude gradient of a tropical island stream. Freshwater Biol. 47: 377-390.

Martínez-Mayen, M. \& R. Román-Contreras. 2000. Aspects of the reproduction of Atya margaritacea A. Milne-Edwards, 1864 (Decapoda, Atyidae) in a population from the Mexican Pacific. Crustaceana 73: 913-923.

Martínez-Mayen, M., R. Román-Contreras, A. RochaRamírez \& S. Chazaro-Olvera. 2000. Relative growth of Atya Margaritacea A. Milne-Edwards, 1864 (Decapoda, Atyidae) from the southern pacific coast of Mexico. Crustaceana 73: 525-534.
Milne-Edwards, A. 1864. Révision des Crustacés Macroures de la Famille des Atyoidées. Ann. Soc. Entomol. France 4: 145-152 plate 3.

Pringle, C.M., G.A. Blake, A.P. Covich, K.M. Buzby \& A. Finley. 1993. Effects of omnivorous shrimps in a mountain tropical stream: sediment removal, disturbance of sessile invertebrates and enhancement of understory algal biomass. Oecologia 93: 1-11.

Pringle, C.M. 1996. Atyid shrimp (Decapoda:Atyidae) influence spatial heterogeneity of algal communities over different scales in tropical montane streams, Puerto Rico. Freshwater Biol. 35: 125-140.

Pringle, C.P., M.C. Freeman \& B.J. Freeman. 2000. Regional effects of hydrologic alterations on riverine macrobiota in the New World: tropical-temperate comparisons. BioScience 50: 807-823.

Ricker, W.E. 1975. Computation and interpretation of biological statistics of. fish populations. Bull. Fish Res. Board of Canada 191: 1-382.

Roverso, E.A., V.L. Lobao, J.V. Lombardi \& E. Hortensio. 1993. Relacoes biométricas em Macrobrachium acanthurus Wiegmann, 1836 (Decapoda, Palaemonidae) da Estação Ecológica da Juréia-SP. Bol. Inst. de Pesca 20: 1-6.

Villalobos, F.A. 1943. Estudio morfológico de Atya scabra (Crust. Decap.). Tesis de Maestría, Facultad de Ciencias, UNAM, México D.F., México.

Villalobos, F.A. 1959. Contribución al Conocimiento de los Atyidae de México. II (Crustácea, Decapoda): Estudio de algunas especies del género Potimirim (=Ortmannia), con descripción de una especie nueva en Brasil. An. del Inst. de Biol. UNAM, México 30: 269-330. 